\title{
A New Proof of an Inequality for the Logarithm of the Gamma Function and Its Sharpness
}

\author{
Mansour Mahmoud ${ }^{1,2, *}$ \\ ${ }^{1}$ Department of Mathematics, Faculty of Science, King Abdulaziz University, P. O. Box 80203, Jeddah 21589, Saudi Arabia \\ ${ }^{2}$ Department of Mathematics, Faculty of Science, Mansoura University, ansoura 35516, Egypt \\ *Corresponding author: mansour@mans.edu.eg
}

Received July 03, 2014; Revised September 01, 2014; Accepted September 08, 2014

\begin{abstract}
In the paper, the author shows that the partial sums $\gamma+\ln x+\frac{1}{2 x}-\sum_{k=1}^{m} \frac{B_{2 k}}{2 k x^{2 k}} ; m=1,2$, are alternatively larger and smaller than the generalized Euler's harmonic numbers $H(x)=\psi(x+1)+\gamma$ with sharp bounds, where $\gamma$ is the Euler's constant, $B_{i}{ }^{\prime} s$ are the Bernoulli numbers and $\psi$ is the digamma function.
\end{abstract}

Keywords: Euler constant, $\psi$-function, harmonic numbers, inequalities, asymptotic expansion, sharp bounds

Cite This Article: Mansour Mahmoud, "A New Proof of An Inequality for the Logarithm of the Gamma Function and Its Sharpness." Turkish Journal of Analysis and Number Theory, vol. 2, no. 4 (2014): 147-151. doi: 10.12691/tjant-2-4-8.

\section{Introduction}

Euler, in his "Institutiones calculi differentialis" [10], introduced the concept of inexplicable functions. These functions were appeared originally as functions in the positive integers of one symbol or more. He presented the following examples of the inexplicable functions:

$$
\begin{aligned}
& F(x)=1 \times 2 \times 3 \times \ldots \times x \\
& H(x)=\frac{1}{1}+\frac{1}{2}+\frac{1}{3}+\ldots+\frac{1}{x}, \\
& \frac{1}{1^{n}}+\frac{1}{2^{n}}+\frac{1}{3^{n}}+\ldots+\frac{1}{x^{n}} \\
& 1+\frac{a-b}{a+2 b}+\frac{a-2 b}{a+3 b}+\frac{a-3 b}{a+4 b}+\ldots+\frac{a-(x-1) b}{a+(x+1) b}
\end{aligned}
$$

where there is no necessity for $\mathrm{x}$ to be an integer. The first function $F(x)$ generalizes the factorial function and the second one $H(x)$ generalizes the harmonic numbers

$$
H_{n}=\sum_{k=1}^{n} \frac{1}{k} ; n \in N
$$

which are partial sums of the harmonic series. The function $H(x)$ can be defined by the definite integral

$$
H(x)=\int_{0}^{1} \frac{1-y^{x}}{1-y} d y .
$$

We will call the function $H(x)$, Euler's generalized Harmonic numbers. The recurrence relation of $H(x)$ is given by

$$
H(x)=H(x-1)+\frac{1}{x}
$$

and its reflection relation is

$$
H(1-x)=H(x)+\pi \cot (\pi x)+\frac{2 x-1}{x(1-x)} .
$$

The multiplication formula is given by

$$
\begin{aligned}
& H(n x)=H(x)+H(x-1 / n)+H(x-2 / n) \\
& +\ldots+H(x-(n-1) / n)+\ln n ; n \in N .
\end{aligned}
$$

The function $H(x)$ is related to the Euler's constant $\gamma$ by the relation

$$
\gamma=\int_{0}^{1} H(x) d x
$$

and its relation with the digamma function $\psi(x)$ (the logarithmic derivative of the gamma function) is

$$
\psi(x+1)=-\gamma+H(x) .
$$

There are many other generalizations of the harmonic numbers all of them depend only on the positive integers see $[3,7,8,11,12,13,17,30,31]$. Also, there are many estimations of the harmonic numbers $H_{n}$ see $[4,5,6,9,18,22,25,26,27,28,32,33]$.

A function $\mathrm{f}$ is said to be completely monotonic on an interval $\mathrm{J}$ if 


$$
(-1)^{n} f^{(n)}(x) \geq 0 \forall x \in J \text { and } n=0,1,2, \ldots .
$$

If the inequality (5) is strict $\forall x \in J$ and all $n=0,1,2, \ldots$, then $\mathrm{f}$ is said to be strictly completely monotonic on the interval J.

In [1], Alzer proved that the functions

$$
\begin{aligned}
& F_{m}(x)=\ln \Gamma(x)-\left(x-\frac{1}{2}\right) \ln x \\
& +x-\ln \sqrt{2 \pi}-\sum_{j=1}^{2 m} \frac{B_{2 j}}{2 j(2 j-1) x^{2 j-1}}
\end{aligned}
$$

and

$$
\begin{aligned}
& G_{m}(x)=\ln \Gamma(x)-\left(x-\frac{1}{2}\right) \ln x \\
& -x+\ln \sqrt{2 \pi}-\sum_{j=1}^{2 m+1} \frac{B_{2 j}}{2 j(2 j-1) x^{2 j-1}}
\end{aligned}
$$

For $m=0,1,2, \ldots$, are completely monotonic on $(0, \infty)$. This means that the functions $-F_{m}{ }^{\prime}(x)$ and $-G_{m}{ }^{\prime}(x)$ are also are completely monotonic on $(0, \infty)$ see [15]. These complete monotonicity have been repeated in [16] and [23]. These results can also be found in the survey [29]. From the complete monotonicity of $-F_{m}{ }^{\prime}(x)$ and $-G_{m}{ }^{\prime}(x)$, we get the following double inequality

$$
\begin{aligned}
& -\sum_{k=1}^{2 m+1} \frac{B_{2 k}}{2 k x^{2 k}}<\psi(x+1)-\ln x-\frac{1}{2 x} \\
& <-\sum_{k=1}^{2 m} \frac{B_{2 k}}{2 k x^{2 k}}, \forall x \in(0, \infty) ; m=0,1,2, \ldots .
\end{aligned}
$$

In this paper, we will present a new proof of the double inequality (6), using Artin's technique [2] and we will use a method due to Mortici [24] to prove that the bounds in (6) are the best possible. Also, we will provide new proof of the complete monotonicity of the two Functions $F_{m}(x)$ and $G_{m}(x)$.

\section{Main Results}

\section{Theorem 1.}

For $\mathrm{x}>0, m=0,1,2, \ldots$,

$$
\sum_{k=1}^{2 m} \frac{B_{2 k}}{2 k x^{2 k}}<\gamma-H(x)+\ln x+\frac{1}{2 x}<\sum_{k=1}^{2 m+1} \frac{B_{2 k}}{2 k x^{2 k}}
$$

with sharp bounds.

\section{Proof}

Consider the function

$$
r(x)=\gamma-H(x)+\ln x+\frac{1}{2 x} ; x>0 .
$$

Then using the recurrence relation (3), we get

$$
r(x)-r(x+1)=\frac{2 x+1}{2 x(x+1)}-\ln \left(\frac{x+1}{x}\right) .
$$

If we define the following function

$$
g(x)=r(x)-r(x+1),
$$

then

$$
r(x)=\sum_{n=0}^{\infty} g(x+n) .
$$

We can express the function $g(x)$ by the integral representation

$$
g(x)=\int_{0}^{1} \frac{1 / 2-v}{(x+v)^{2}} d v
$$

and hence

$$
r(x)=\sum_{n=0}^{\infty} \int_{0}^{1} \frac{1 / 2-v}{(x+n+v)^{2}} d v .
$$

By considering the known discontinuous function

$$
K(v)=\left\{\begin{array}{cc}
\frac{1}{2}-v & 0<v<1 \\
0 & v=0 \\
\text { periodic of period } 1 & \text { otherwise }
\end{array}\right.
$$

we get

$$
\begin{aligned}
& r(x)=\sum_{n=0}^{\infty} \int_{n}^{n+1} \frac{K(v)}{(x+v)^{2}} d v \\
& =\lim _{n \rightarrow \infty} \int_{0}^{n} \frac{K(v)}{(x+v)^{2}} d v .
\end{aligned}
$$

The oscillating of the function $\frac{K(v)}{(x+v)^{2}}$ and $\lim _{v \rightarrow \infty} \frac{K(v)}{(x+v)^{2}}=0$ provided us by the existence of the integration

$$
r(x)=\int_{0}^{\infty} \frac{K(v)}{(x+v)^{2}} d v .
$$

In [2], Artin introduced the functions

$$
\begin{aligned}
& K_{2 n}(v)=2(-1)^{n-1} \sum_{k=1}^{\infty} \frac{\cos (2 k \pi v)}{(2 k \pi)^{2 n}} \\
& K_{2 n+1}(v)=2(-1)^{n} \sum_{k=1}^{\infty} \frac{\sin (2 k \pi v)}{(2 k \pi)^{2 n-1}},
\end{aligned}
$$

Where $-K_{1}(v)$ is the Fourier series $[20,21]$ of the function $K(v)$. He showed that the series $K_{n}(v)$ is absolutely and uniformly convergent for all $\mathrm{v}$ with $\mathrm{n}=2,3, \ldots$ and the series is uniformly convergent in every closed interval has no integer for $n=1$. Also,

$$
\frac{d}{d v} K_{n+1}(v)=K_{n}(v)
$$

for all $\mathrm{v}$ when $\mathrm{n}=2,3, \ldots$ and for nonintegral $\mathrm{v}$ when $\mathrm{n}=1$. 
Now by repeated the integrations by parts of (10), we obtain

$$
\begin{aligned}
& r(x)=\frac{K_{2}(0) 1 !}{x^{2}}+\frac{K_{3}(0) 2 !}{x^{3}}+\ldots+ \\
& \frac{K_{n}(0)(n-1) !}{x^{n}}-\int_{0}^{\infty} \frac{K_{n}(v) n !}{(x+v)^{n+1}} d v .
\end{aligned}
$$

But

$$
\begin{aligned}
& \frac{K_{n}(0)(n-1) !}{x^{n}}-\int_{0}^{\infty} \frac{K_{n}(v) n !}{(x+v)^{n+1}} d v \\
& =\int_{0}^{\infty} \frac{\left(K_{n}(0)-K_{n}(v)\right) n !}{(x+v)^{n+1}} d v .
\end{aligned}
$$

If $\mathrm{n}$ is even, the $K_{n}(0)-K_{n}(v)$ has the same sign as $K_{n}(0)$ for all $\mathrm{v}$ and thus the integral (15) has this sign. Also,

$$
K_{2 n+1}(0)=0 \text { and } K_{2 n}(0)=\frac{B_{2 n}}{(2 n) !}, \forall n .
$$

Then the signs of $K_{2 n}(0)$ alternate between minus and plus. Then the function $r(x)$ lies between any two successive partial sums

$$
\frac{K_{2}(0) 1 !}{x^{2}}+\frac{K_{4}(0) 3 !}{x^{4}}+\ldots+\frac{K_{2 n}(0)(2 n-1) !}{x^{2 n}}
$$

In other words, for every $\mathrm{n}$, there exists a number $\theta_{n}$ satisfies

$$
\begin{aligned}
& r(x)=\frac{B_{2}}{2 x^{2}}+\frac{B_{4}}{4 x^{4}}+\ldots+\frac{B_{2 n-2}}{(2 n-2) x^{2 n-2}} \\
& +\frac{B_{2 n}}{2 n x^{2 n}} \theta_{n}, 0<\theta_{n}<1
\end{aligned}
$$

and hence

$$
\sum_{k=1}^{2 m} \frac{B_{2 k}}{2 k x^{2 k}}<\gamma-H(x)+\ln x+\frac{1}{2 x}<\sum_{k=1}^{2 m+1} \frac{B_{2 k}}{2 k x^{2 k}} .
$$

Now, we will prove the sharpness of the bounds in (19). By the definition of the asymptotic expansion [14], the expansion of a function $F(x)$ of the form

$$
F(x)=g(x)+a_{0}+\frac{a_{1}}{x}+\frac{a_{2}}{x^{2}}+\ldots
$$

satisfies for every fixed $\mathrm{k}$, that

$$
\lim _{x \rightarrow \infty} x^{k}\left[F(x)-\left(g(x)+a_{0}+\frac{a_{1}}{x}+\ldots+\frac{a_{k}}{x^{k}}\right)\right]=0 .
$$

Then

$$
\begin{aligned}
& \lim _{x \rightarrow \infty} x^{2 m}\left[H(x)-\ln x-\gamma-\frac{1}{2 x}+\sum_{k=1}^{2 m-1} \frac{B_{2 k}}{2 k x^{2 k}}\right] \\
& =-\frac{B_{2 m}}{2 m}, m=1,2,3, \ldots
\end{aligned}
$$

If we have other constants $c_{2}, c_{4}, c_{6}, \ldots$ have the property that for all $n \in N$

$$
\begin{aligned}
& -\frac{c_{2}}{x^{2}}-\frac{c_{4}}{x^{4}}-\frac{c_{6}}{x^{6}}<H(x)-\ln x-\gamma-\frac{1}{2 x}<-\frac{c_{2}}{x^{2}}-\frac{c_{4}}{x^{4}}, \\
& -\frac{c_{2}}{x^{2}}-\frac{c_{4}}{x^{4}}-\frac{c_{6}}{x^{6}}-\frac{c_{8}}{x^{8}}-\frac{c_{10}}{x^{10}}<H(x)-\ln x-\gamma-\frac{1}{2 x} \\
& <-\frac{c_{2}}{x^{2}}-\frac{c_{4}}{x^{4}}-\frac{c_{6}}{x^{6}}-\frac{c_{8}}{x^{8}}, \\
& -\frac{c_{2}}{x^{2}}-\frac{c_{4}}{x^{4}}-\frac{c_{6}}{x^{6}}-\frac{c_{8}}{x^{8}}-\frac{c_{10}}{x^{10}}-\frac{c_{12}}{x^{12}}-\frac{c_{14}}{x^{14}}< \\
& H(x)-\ln x-\gamma-\frac{1}{2 x} \\
& <-\frac{c_{2}}{x^{2}}-\frac{c_{4}}{x^{4}}-\frac{c_{6}}{x^{6}}-\frac{c_{8}}{x^{8}}-\frac{c_{10}}{x^{10}}-\frac{c_{12}}{x^{12}},
\end{aligned}
$$

etc. These inequalities give us that

$$
\begin{gathered}
\lim _{x \rightarrow \infty} x^{2}\left[H(x)-\ln x-\gamma-\frac{1}{2 x}\right]=-c_{2}, \\
\lim _{x \rightarrow \infty} x^{4}\left[H(x)-\ln x-\gamma-\frac{1}{2 x}+\frac{c_{2}}{x^{2}}\right]=-c_{4}, \\
\lim _{x \rightarrow \infty} x^{6}\left[H(x)-\ln x-\gamma-\frac{1}{2 x}+\frac{c_{2}}{x^{2}}+\frac{c_{4}}{x^{4}}\right]=-c_{6},
\end{gathered}
$$

the relations (20) and (21), gives us that

$$
c_{2 l}=\frac{B_{2 l}}{2 l}, \forall l \in N
$$

Then the choice of the constants $\frac{B_{2 k}}{2 k}$ in the inequality (7) is the best one. To complete our results, we need to prove that the constant $1 / 2$ in the the function $H(x)-\ln x-\gamma-\frac{1}{2 x}$ can not be improved by any method whatsoever. Consider the function

$$
Z(x)=H(x)-\ln x-\gamma-\frac{A}{x},
$$

then

$$
Z(x)-Z(x+1)=\frac{-x-A}{x(x+1)}+\ln \left(\frac{x+1}{x}\right) .
$$

Now, let

$$
V(x)=\frac{-x-A}{x(x+1)}+\ln \left(\frac{x+1}{x}\right) ; x>0
$$

then

$$
V^{\prime}(x)=\frac{A(2 x+1)-x}{x^{2}(x+1)^{2}} .
$$

The The function $V(x)$ will be increasing if

$$
A>\frac{x}{2 x+1}=v(x)
$$


and the function $v(x)$ is increasing function with $\lim _{x \rightarrow \infty} v(x)=1 / 2$. So, the best choice of $\mathrm{A}$ is $1 / 2$. Also, the function $v(x)$ is increasing with limit tends to zero as $\mathrm{x} \rightarrow \infty$, then

$$
V(x)<0
$$

Hence

$$
Z(x)<Z(x+n), n \in N
$$

As $n \rightarrow \infty$, we get

$$
Z(x)<0 \text { or } H(x)-\ln x-\gamma<\frac{1}{2 x} .
$$

with sharp bound. Now, consider the function

$$
W(x)=H(x)-\ln x-\gamma-\frac{1}{2 x}+\frac{B}{x^{2}},
$$

then

$$
\begin{aligned}
& W(x+1)-W(x)= \\
& \frac{-2 B(1+2 x)+x+3 x^{2}+2 x^{3}}{2 x^{2}(x+1)^{2}}+\ln \left(\frac{x}{x+1}\right) .
\end{aligned}
$$

Let

$$
T(x)=\frac{-2 B(1+2 x)+x+3 x^{2}+2 x^{3}}{2 x^{2}(x+1)^{2}}+\ln \left(\frac{x}{x+1}\right) ; x>0
$$

then

$$
T^{\prime}(x)=\frac{4 B\left(1+3 x+3 x^{2}\right)-x(1+x)}{3 x^{3}(x+1)^{3}}
$$

The function $T(x)$ will be increasing if

$$
B>\frac{x(1+x)}{4\left(1+3 x+3 x^{2}\right)}=t(x)
$$

and the function $t(x)$ is increasing function with $\lim _{x \rightarrow \infty} t(x)=1 / 12$. So, the best choice of $\mathrm{B}$ is $1 / 12$. Also, the function $T(x)$ is increasing with limit tends to zero as $x \rightarrow \infty$, then

$$
T(x)<0
$$

Hence

$$
W(x+n)<W(x), n \in N .
$$

As $n \rightarrow \infty$, we get

$$
W(x)>0 \text { or } H(x)-\ln x-\gamma>\frac{1}{2 x}-\frac{1}{12 x^{2}} .
$$

Hence

$$
\frac{1}{2 x}-\frac{B_{2}}{x^{2}}<H(x)-\ln x-\gamma<\frac{1}{2 x}
$$

with sharp bounds.
As a special case we get the following result [19]

\section{Corollary 2.1.}

For any natural number $n \in N$,

$$
\begin{aligned}
& \frac{1}{2 n}-\sum_{k=1}^{2 m+1} \frac{B_{2 k}}{2 k n^{2 k}}<H_{n}-\ln n-\gamma \\
& <\frac{1}{2 n}-\sum_{k=1}^{2 m} \frac{B_{2 k}}{2 k n^{2 k}} m=0,1,2,3, \ldots
\end{aligned}
$$

with sharp bounds.

Now, we will present a new proof for the complete monotonicity of the functions $F_{m}(x)$ and $G_{m}(x)$.

\section{Lemma 2.2}

For $\mathrm{m}=0,1,2, \ldots$, the functions

$$
\begin{aligned}
& F_{m}(x)=\ln \Gamma(x)-\left(x-\frac{1}{2}\right) \ln x+x \\
& -\ln \sqrt{2 \pi}-\sum_{j=1}^{2 m} \frac{B_{2 j}}{2 j(2 j-1) x^{2 j-1}}
\end{aligned}
$$

and

$$
\begin{aligned}
& G_{m}(x)=-\ln \Gamma(x)+\left(x-\frac{1}{2}\right) \ln x-x \\
& +\ln \sqrt{2 \pi}+\sum_{j=1}^{2 m+1} \frac{B_{2 j}}{2 j(2 j-1) x^{2 j-1}}
\end{aligned}
$$

are completely monotonic on $(0, \infty)$.

\section{Proof}

Using the relations (15), (17) and (16) at $n=4 m+2$, we get

$$
\begin{aligned}
& r(x)=\sum_{j=1}^{2 m} \frac{B_{2 j}}{2 j x^{2 j}}+ \\
& \int_{0}^{\infty} \frac{\left(K_{4 m+2}(0)-K_{4 m+2}(v)\right)(4 m+2) !}{(x+v)^{4 m+3}} d v
\end{aligned}
$$

and hence

$$
\begin{aligned}
& (-1)^{r} \frac{d^{r}}{d x^{r}}\left(r(x)-\sum_{j=1}^{2 m} \frac{B_{2 j}}{2 j x^{2 j}}\right)= \\
& \int_{0}^{\infty} \frac{\left(K_{4 m+2}(0)-K_{4 m+2}(v)\right)(4 m+2+r) !}{(x+v)^{4 m+3+r}} d v, \\
& r=1,2,3, \ldots
\end{aligned}
$$

But for $\mathrm{m}=0,1,2,3, \ldots$, the $K_{4 m+2}(0)-K_{4 m+2}(v)$ has the same sign as $K_{4 m+2}(0)$ for all $\mathrm{v}$ and thus the integral (24) has this sign. Using the relation (11), we obtain

$$
K_{4 m+2}(0)=2(-1)^{2 m} \sum_{k=1}^{\infty} \frac{1}{(2 k \pi)^{2 n}}>0
$$

and hence

$$
(-1)^{r} \frac{d^{r}}{d x^{r}}\left(r(x)-\sum_{j=1}^{2 m} \frac{B_{2 j}}{2 j x^{2 j}}\right)>0, r=1,2,3, \ldots
$$

But 


$$
\frac{d}{d x} F_{m}(x)=-\left(r(x)-\sum_{j=1}^{2 m} \frac{B_{2 j}}{2 j x^{2 j}}\right),
$$

then

$$
(-1)^{r} \frac{d^{r}}{d x^{r}} F_{m}(x)>0, r=0,1,2, \ldots
$$

Similarly, we can prove the complete monotonicity for $G(x)$ by replacing $n$ by $4 \mathrm{~m}+4$.

\section{Remark 1.}

Series (17) is divergent so we can not take the limit as n tends to $\infty$ but the relation (18) provided us by approximations of the function $H(x)$ or any finite order. For example, if $\mathrm{n}=5$ we obtain the following approximation:

$$
\begin{aligned}
& H(x)=\gamma+\ln x+\frac{1}{2 x}-\frac{1}{12 x^{2}}+\frac{1}{120 x^{4}} \\
& -\frac{1}{252 x^{6}}+\frac{1}{240 x^{8}}-\frac{\theta}{132 x^{10}}, 0<\theta<1 .
\end{aligned}
$$

Remark 2.

The formula (10) implies the the function $r(x)$ is completely monotonic on $(0, \infty)$, that is

$$
(-1)^{r} r^{(k)}(x) \geq 0, \forall k \in N ; x \in(0, \infty) .
$$

\section{Acknowledgement}

The author is very grateful to Prof. Feng Qi at Tianjin Polytechnic University in China for valuable comments and useful suggestions, which greatly helped improve the presentation and the quality of the paper.

\section{References}

[1] Alzer, H., "On some inequalities for the gamma and psi functions," Mathematics of Computation, 66, no. 217, 373-389, 1997.

[2] Artin, E., The Gamma function, translated by M. Butler, Holt, Rinehart and Winston, New York, 1964.

[3] Benjamin, A. T., Gaebler, D. and Gaebler, R., "A combinatorial approach to hyperharmonic numbers", Integers, 3: A15, 2003.

[4] Chen, C.-P. and Qi, F., "The best bounds of the n-th harmonic number", Glob. J. Appl. Math. Math. Sci. 1, no. 1, 41-49, 2008.

[5] Chen, C.-P., "Inequalities for Euler-Mascheroni constant," Appl. Math. Lett. 23, 161-164, 2010.

[6] Guo, B.-N. and Qi, F., "Sharp bounds for harmonic numbers", Applied Mathematics and Computation, Vol. 218, 3, 991-995, 2011.

[7] Chu, W., "Harmonic number identities and Hermite-Padé approximations to the logarithm function, J. Approx. Theory, 137, 42-56, 2005.
[8] Coffey, M. W. and Lubbers, N., "On generalized harmonic number Sums", Applied Mathematics and Computation, Vol. 217, Issue 2, 689-698, 2010.

[9] DeTemple, D. W., "A quicker convergence to Euler's Constant", The Amer. Math. Monthly 100, 468-470, 1993.

[10] Euler, L., "Institutiones calculi differentialis", St. Petersburg, 1755.

[11] Gertsch, A., "Generalized harmonic numbers", C. R. Acad. Sci. Paris, Sér. I Math. 324, no. 1, 7-10, 1997.

[12] Gessel, I. M., "On Miki's identity for Bernoulli numbers", J. Number Theory, 110, 75-82, 2005.

[13] Ken, K., "On 3-adic valuations of generalized harmonic numbers", Integers, Vol. 12, Issue 2, 311-319, 2012.

[14] Knopp, K., Theory and application of infinite series, Dover, New York, 1990.

[15] Koumandos, S., "Remarks on some completely monotonic functions", J. Math. Anal. Appl. 324, 1458-1461, 2006.

[16] Koumandos, S. and Pedersen, H. L., "Completely monotonic functions of positive order and asymptotic expansions of the logarithm of Barnes double gamma function and Euler's gamma function", J. Math. Anal. Appl. 355, no. 1, 33-40, 2009.

[17] Kronenburg, M. J., "Some generalized harmonic number identities", arXiv: 1103.5430v2, 2012.

[18] Mansour, M., "On quicker convergence towards Euler's constant", J. Comput. Anal. Appl. 17, No. 4, 632-638, 2014.

[19] Mahmoud, M., "On the bounds of Euler's constant $\gamma$, Life Sci J, 11 (9): 617-621, 2014.

[20] Mishra, V. N. and Mishra, L. N., "Trigonometric Approximation of Signals (Functions) in $\mathrm{L}_{\mathrm{p}}$-Norm", Int. Journal of Contemp. Math. Sciences, Vol. 7, no. 19, 909-918, 2012.

[21] Mishra, V. N., Khatri, K. and Mishra, L. N., "Using Linear Operators to Approximate Signals of $\operatorname{Lip}(\alpha, p),(\mathrm{p} \geq 1)$-Class, Filomat 27: 2, 353-363, 2013.

[22] Mortici, C., "Fast convergences towards Euler-Mascheroni constant", Computational and Appl. Math., Vol. 29, N. 3, 479-491, 2010.

[23] Mortici, C., "Very accurate estimates of the polygamma functions", Asympt. Anal. 68, no. 3, 125-134, 2010.

[24] Mortici, C., "A new representation formula for the factorial function", Thai Journal of Mathematics, Vol. 8, no. 2, 249-254, 2010.

[25] Qi, F., Cui, R.-Q., Chen, C.-P. and Guo, B.-N., "Some completely monotonic functions involving polygamma functions and an application", Journal of Mathematical Analysis and Applications 310, no. 1, 303-308, 2005.

[26] Qi, F. and Luo, Q.-M., "Bounds for the ratio of two gamma functions--From Wendel's and related inequalities to logarithmically completely monotonic functions", Banach Journal of Mathematical Analysis 6, no. 2, 132-158, 2012.

[27] Qi, F. and Luo, Q.-M., "Bounds for the ratio of two gamma functions: from Wendel's asymptotic relation to ElezovićGiordano-Pečarić's theorem, Journal of Inequalities and Applications 2013, 2013: 542, 20 pages.

[28] Guo, B.-N. and Qi, F., "Sharp inequalities for the psi function and harmonic numbers", Analysis--International mathematical journal of analysis and its applications 34,2014 , in press.

[29] Qi, F., "Bounds for the ratio of two gamma functions", J. Inequal. Appl., Vol. 2010, Article ID 493058, 84 pages.

[30] Cheon, Gi-S., and El-Mikkawy, M. A., "Generalized harmonic numbers with Riordan arrays", J. Number Theory, 128, 413-425, 2008.

[31] Santmyer, J. M., "A Stirling like sequence of rational numbers", Discrete Math. 171, 229-239, 1997.

[32] Tóth, L., Problem E3432, Amer. Math. Monthly, 98 (3), 264, 1991.

[33] Young, R. M., "Euler's constant", Math. Gazette 75, Vol 472, 187-190, 1991. 